\title{
SUR LA FONCTION DU CORPS PARABASAL DE GIARDIA
}

\section{Par G. LAVIER}

Kofoid et Christiansen (1915) ont appliqué le nom de corps parabasaux créé par Janicki pour un organelle de Trichomonas aux masses sidérophiles que présentent les espèces du genre Giardia, en dessous des noyaux et en arrière des deux filaments axiaux ; ce sont les " rätselhafte Körper " de Bensen. Quoique l'assimilation de ces masses au corps parabasal des trichomonades soit discutable, le nom donné par Kofoid et Christiansen a néanmoins prévalu et est constamment employé par tous les auteurs.

La nature et la fonction de ces corps furent longtemps considérées comme totalement inconnues. Alexeieff (1917) fit du corps parabasal des trichomonades une formation mitochondriale à sécrétion glycoplastique ; étendant cette vue aux autres flagellés, il considère le corps parabasal des Giardia comme ayant un rôle de sécrétion du glycogène.

Bœek (1919), après lui, étudia le corps parabasal de Giardia microti : il le considère comme situé dans une région définie du cytoplasme (parabasal plasm) paraissant limitée par une sorte de membrane (membrane-like structure). Reprenant les vues d'Alexeieff, il considère aussi le corps parabasal comme sécrétant le glycogène, car il donne la réaction acajou avec l'iode, mais à la vérité de façon inconstante ; il s'éloigne toutefois d'Alexeieff en ce qu'il considère le corps parabasal comme d'origine cytoplasmique et non pas d'origine nucléaire.

En ce qui concerne l'assertion d'Alexeieff, à aucun moment l'auteur n'explique quelles en sont les bases. Bœck examine les Giardia dans la solution de Lugol après fixation au Schaudinn et passage à l'alcool à $95^{\circ}$.

J'ai eu l'occasion d'examiner de nombreuses formes végétatives de Giardia intestinalis, de G. muris, de G. varani en les fixant directement à la solution de Lugol. Je n'ai jamais vu que les corps parabasaux se colorassent en acajou ; ils prennent en réalité une teinte jaune d'autant plus foncée que la teneur en iode est plus forte ; cette teinte est la même que celle des noyaux. Quant au cytoplasme, parfois il se colore en jaune très pâle, parfois il donne la réaction du glycogène, mais dans ce dernier cas, la teinte acajou est répartie dans toute la masse du protozoaire ; si elle peut parfois paraître plus intense dans la partie inférieure de l'animal c'est uniquement parce qu'il présente là son épaisseur maxima, mais le corps

Annales de Parasitologie, T. I, No 4 . - Octobre 1923. 
parabasal tranche alors par sa réfringence et sa couleur plus claire. Quant au " parabasal plasm " et à cette apparence de membrane que signale Bœck il ne m'a jamais été possible de les voir ; le corps parabasal se présente au contraire dans le cytoplasme comme une enclave nette et sans contour analogue aux corps sidérophiles d'Entamœba dysenteriæ dans les formes kystiques.

Le liquide de Lugol ne permettant qu'un examen extemporané, je me suis adressé à deux méthodes de coloration élective du glycogène permettant de faire des préparations stables. J'ai employé successivement la méthode de Best au carmin et celle de VastariniCresi à la fuchsine résorcinée. La fixation dans les deux cas était faite avec le liquide de Carnoy et la conservation avant la coloration dans l'alcool à $95^{\circ}$ saturé de glucose. Ainsi était évité la dissolution possible du glycogène en milieu aqueux.

Les deux méthodes m'ont donné des résultats identiques à ceux fournis par le Lugol. Le glycogène est coloré en carmin par la première méthode, en rouge vif par la seconde. Comme avec l'iode, on trouve des individus colorés de façon très pâle et d'autres plus intensivement, mais on aperçoit toujours non colorées 3 masses : les deux noyaux et le corps parabasal. Le glycogène plus ou moins abondant est toujours réparti dans tout le cytoplasme, généralement de façon homogène, donnant alors une teinte générale carmin ou rouge vif, mais parfois, dans les individus qui présentent fortement la réaction, on peut voir le cytoplasme, dans toutes ses parties, bourré de granulations glycogéniques; celles-ci ne sont pas particulièrement massées au voisinage des corps parabasaux.

Ainsi l'opinion d'Alexeieff et de Bœck n'est pas soutenable. Le corps parabasal de Giardia ne paraît pas plus que tout autre organelle de ce protozoaire avoir de rapport avec la sécrétion glycogénique, et sa fonction de même qu'auparavant nous demeure inconnue.

\section{BiBLIOGRAPHIE}

AlexerefF (A.). - Mitochondries et corps parabasal chez les Flagellés. Réunion biologique de Petrograd in C. R. Soc. biol., LXXIX, 1917, p. 358.

- Nature mitochondriale du corps parabasal des Flagellés. Ibid., p. 499.

- Sur les mitochondries à fonction glycoplastique. Ibid., p. 510.

- Sur la fonction glycoplastique du kinétoplaste (= kinetonucleus) chez les Flagellés. Ibid., p. 512.

Вовск (W.-C.). - Studies on Giardia microti: The parabasal bodies of Giardia microti. Univ. of California Publ. in Zoology, XIX, 1919, n 3, p. 102.

Laboratoire de Parasitologie de la Faculté de médecine de Paris. 